\title{
GESTURE CLASSIFICATION USING MACHINE LEARNING APPROACH
}

\author{
KETKI P. KSHIRSAGAR ${ }^{1}$, RAJESHREE ROKADE ${ }^{2}$ \\ ${ }^{l}$ E\&TC Department, Vishawakarma Institute of Information Technology, Pune, Maharashtra, India \\ ${ }^{2}$ E\&TC Department, Lokmanya Tilak College of Engineering, Navi Mumbai, Maharashtra, India
}

\begin{abstract}
Gesture classification is vital role to convey information between communications. Classification shows correct path for predict correct communication. Machine learning provide automated improved algorithm in the set of artificial intelligence. In this paper, we proposed machine learning classification approach for hand gesture recognition. In classification system classify the hand gesture images with better accuracy. Proposed system also differentiates different activation functions for non linear transformation of hand gestures as input to complex tasks. Proposed system compares and classifies dataset using convolution neural network algorithm using different activation functions.
\end{abstract}

KEYWORDS: Activation Function, Convolution Neural Network, Hand Gesture, Classification

Received: Jun 08, 2020; Accepted: Jun 28, 2020; Published: Sep 16, 2020; Paper Id.: IJMPERDJUN20201280

\section{INTRODUCTION}

Artificial neural networks tries to behave like brain network and neurons are back bone of network. Using weight, bias and activation function neurons are characterized. So, activation function show important role in neural network characterization. There are ten activation functions are available like binary, linear, sigmoid, Tanh, ReLu, parameterized ReLu, exponential linear, leaky ReLu ,Softmax. With activation function network get complex and powerful for solving any complex problem. In major complex problems ReLu activation function is used because ReLu cannot activate all neurons at the same time. Softmax activation function used in multiclass classification problem because of Softmax is combination of sigmoids. In our proposed system experimented the use of different activation functions in convolution neural network for classification of hand gestures.

The rest of paper is organized as follows: section I introduces related recent work; section II provides proposed convolution neural network based classification system, section III discussed experimental results and section IV provide conclusion and future scope.

\section{Related Recent Work}

Adam Ahmed Qaid Mohammed et.al. [1] proposed deep learning based complex and cluttered environmental function of detecting and classifying hand gestures. The researchers preprocessed in complex and cluttered environment such as high levels occlusion, extreme illumination change in environment, hand variations and low resolution. In his proposed system hand extracted using lightweight convolution neural network. For experimentation they used publicly available data. Datasets includes Egohands, Oxford , 5 signers and Indian classical dance images. Especially the researchers worked on 41000 training static LaRED and Tiny Hand of one and two hand datasets. The proposed system finding hand recognition accuracy using average precision, F1 score 
and average recall parameter. The hand detection accuracy improved upto 94.4\%. Mais Yasen and Shaidah Jusoh proposed hand classification based on artificial neural network. The proposed system majorly focused on overfitting in hand datasets. The researchers reviewed all application of back two to three year gesture recognizing applications [2]. The proposed system worked on low resolution static images [3]. Paulo Trigueiros, Fernando Ribeiro et. al. proposed hand gesture classification used artificial neural network, K-NN, Naïve bayes and SVM algorithm. Artificial neural network algorithm shows better performance between these four algorithms. Artificial neural network classification accuracy improves upto $96.99 \%$ then other algorithm.

Wenjin Tao et. al, proposed sign language alphabet recognition system. Convolution neural network used for recognition purpose[4]. In this proposed system image is captured by Microsoft kinect and recognized alphabets on the basis of multiview argumentation and inference fusion. Becasue of argumentation perspective over fitting is reduced. In this system argumentation algorithm retrieve 3D information in depth images. In proposed system two public benchmark database used for experimentation purpose. In experiment proposed algorithm accuracy efficiency increased upto 93-100 $\%$. The proposed system provide computer interface with artificial neural network and also provide natural interface. In this system they acquire movement based simple database using gesture sensor. Artificial neural network recognition rate is depending on down sampling rate. For experimentation researcher uses three type of dataset like clockwise, anticlockwise and waving data is used with 10 neurons and 5 neurons. In experimentation 10 neuron recognition rate is improved than the 5 neurons [5]. Lionel Pigou et. al.[6] proposed temporal pooling recurrence based convolution system used for gesture recognition. Temporal gesture information shows more discriminative information than general video. The proposed system was work on Jaccard index, precision, recall and error rate for recognition of temporal gesture using convolution neural network and LSTM. With features the experimentation result improved up to 94-95\%.

Kenneth Lai et. al. proposed CNN \&RNN based depth and skeleton based dynamic hand gesture recognition system. Recurrent neural networks provide skeleton information only and CNN used for extracting special information of depth images. Combination of both neural networks used for recognizing video frame images more accurately. The proposed system recognition accuracy improves upto $85.46 \%$ for dynamic hand gesture with 14/28 datasets[7]. The researchers proposed palm and finger recognized computer vision system. K. M. Vamsikrishna et. al classified isolated image using linear discriminate analysis and SVM method. These methods are comparing HMM based sequence classification with CRF based system. In experimentation proposed system shows better result for palm and finger recognition. Experimentation analysis shows better accuracy in all classification techniques of discriminant analysis + HMM,SVM+HMM, DA+ CRF, SVM +CRF and individually discriminant analysis and support vector[8][9]. Juan C. Núñez et. al.[10] proposed CNN and LSTM algorithm which recognized human activity and hand gesture. In proposed system two stage training used. In the proposed system convolution neural network extract 3D skeleton data features and improves two stage training data and avoid overfitting issue. In experimentation proposed system shows remarkable results towards recognition efficiency. Di Wu et. al. proposed novel technique deep dynamic neural network which is based on semi supervised of hidden markov model. Gaussian-Bernouilli Deep network used 3D convolution neural network with jaccard index score of 0.81. Experimental analysis shows skeleton -DBDN validation accuracy 86.3\%, RGB-D-3DCNN validation accuracy 78.7\%, Multimodal late fusion accuracy $87.9 \%$ and multimodal inter fusion improved upto 86.5\%[11]. Cristina Nuzzi et. al. [12]proposedsmart hand gesture recognition system. The system is based on CNN with object detection, which find position of hand in colour images. The system improves incorrect prediction of detector command, which is used for robot performance. The system experimented on 5 diiferent situation like base, light colors, gloves, zoom 
and complete. The system experimentation efficiency improves upto $99.11 \%$.

Guillaume Devineau, Wang Xi et. al., proposed 3D hand gesture recognition approach using deep learning for skeletal data. The proposed system introduced $\mathrm{CNN}$ approach for skeletal images only. For experimentation purpose researchers used 14 and 28 gesture classes. Classification accuracy of respective classes increases up to $91.28 \%$ and 84.35\%[13].The proposed system is based on sEMG based gesture recognition introduced by Istvan Ketyko. The proposed system is based on electrical activity of skin coloured hand part. The researchers proposed domain adaptation based deep learning method for improving enhancement accuracy[14]. Son Thai Ly et. al.[15], proposed emotion based body gesture recognition using deep learning. The proposed system approaches key frame based recognition for time consumption. The experimental result of CNN and LSTM with deep learning approach shows better accuracy than the existing. Kollipara Sai Varun et. al.[16], proposed CNN and back propagation based gesture recognition system. The proposed system worked on training data with CNN approach and experiment testing data with neural network approach and prevented overfitting issue. Novel approach focused on single shot multibox detector deep learning. Peng Liu et. al., experimented on four character hand gestures with different background using 19 layered CNN. Recognition efficiency of SSD algorithm improved $93.8 \%$ to $99.2 \%$. SSD algorithm is more reliable, time efficient and accurate algorithm than the existing one. SSD algorithm shows real time performance with less than 20ms [17].

\section{Proposed Convolution Neural Network Based Classification System}

In proposed system first step is to access standard data if it is .csv or .zip or any other format. In proposed work, Google Colab cloud service and tensor flow used for machine learning for learning application. Input access data convert into training samples, testing samples and validation samples

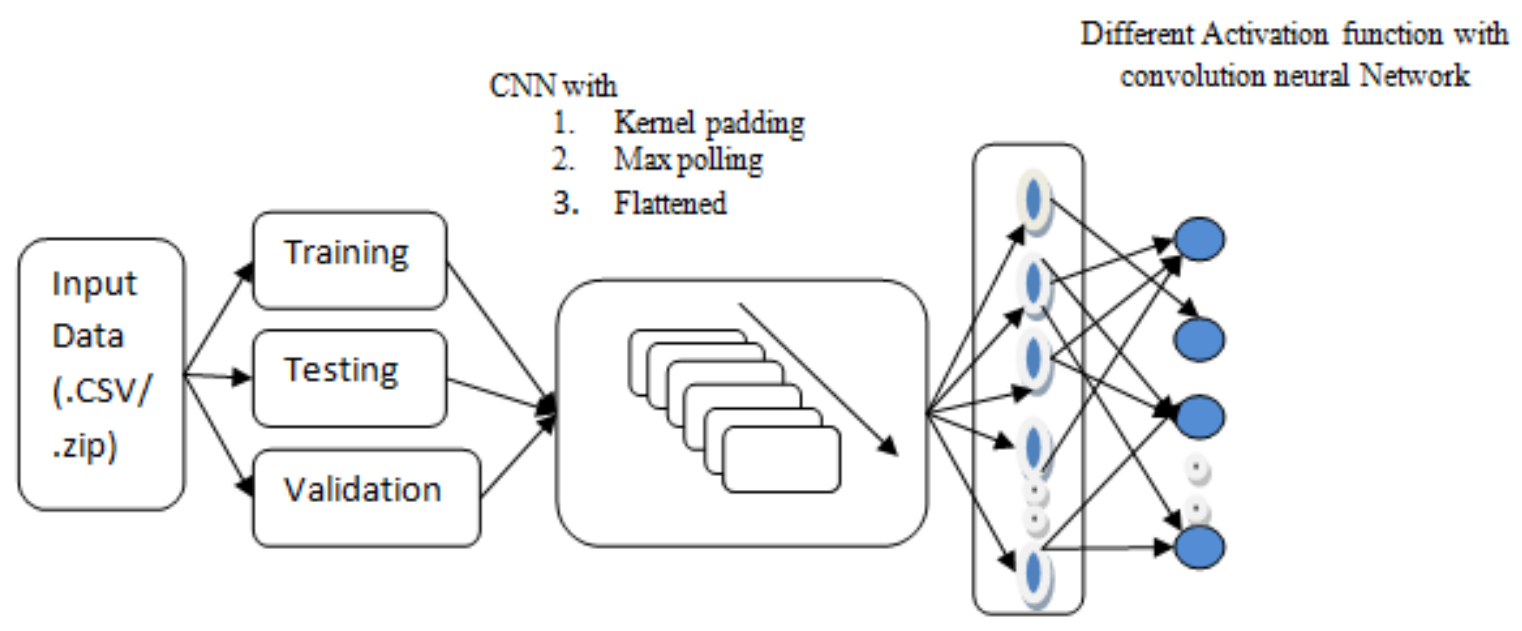

Figure 1: Convolution neural network based classification system

Training samples are used for classification purpose. In classification every sample is processed by kernel padding, max polling and flattened with convolution neural network. All trainable samples are processed by classifier. Proposed system classifies hand gestures very precisely shown in figure 1. After convolution data processed with neural network with different activation functions. Generally ReLu and softmax activation functions are used. In proposed system used Tanh and sigmoid function also. Different activation function shows accuracy and loss result very precise with proposed system. 


\section{Experimental Result}

For experimentation purpose used sign mnist training and sign mnist testing standard dataset. 80\% training samples and $20 \%$ testing samples are used for experimentation. Segregate training samples and convolution neural network model trained with the kernel padding and max polling and flattened the data. In training proposed system observed different activation accuracies and losses. In proposed system sigmoid, ReLu, Tanh, Softmax activation function shows different result accuracy that is shown in figure 2. Combination of ReLu and Softmax activation shows remarkable accuracy with proposed system.

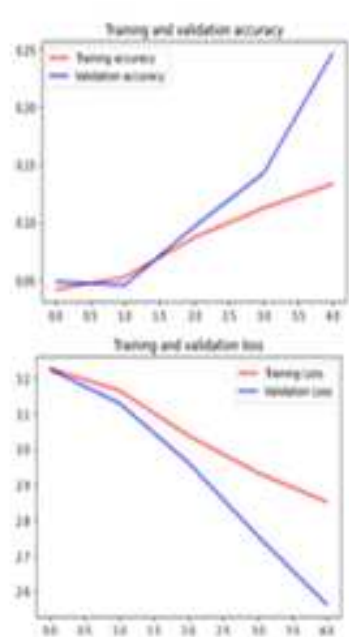

(a)

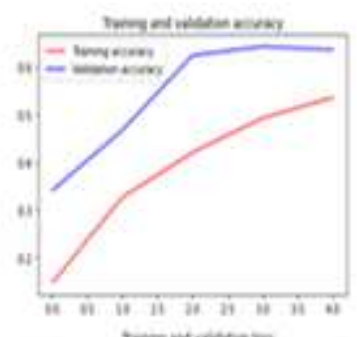

temotiones

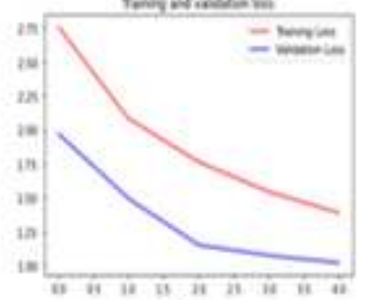

(b)

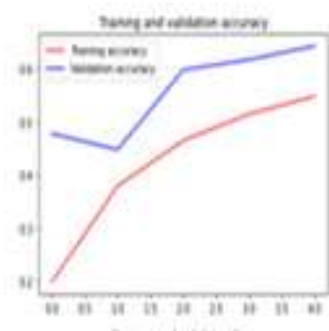

tom wiwisem

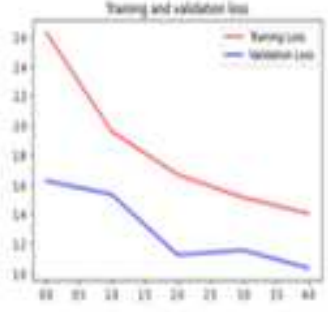

(c)
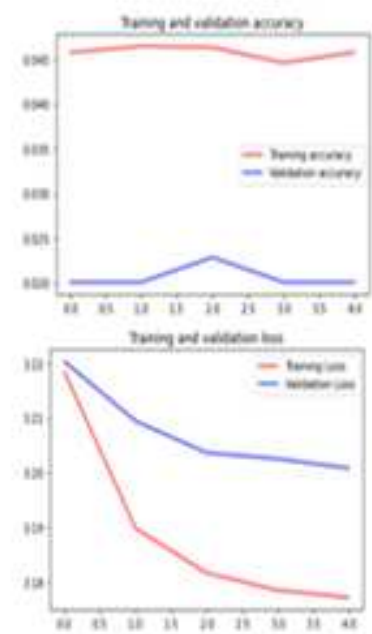

(d)

Figure 2: (a)Sigmoid(b)ReLu(c)Tanh(d)Softmax Activation functions

Convolution neural network classification hand gestures with combination of ReLu and Softmax activation function accuracy after 10 epoch is shown in figure 3 .

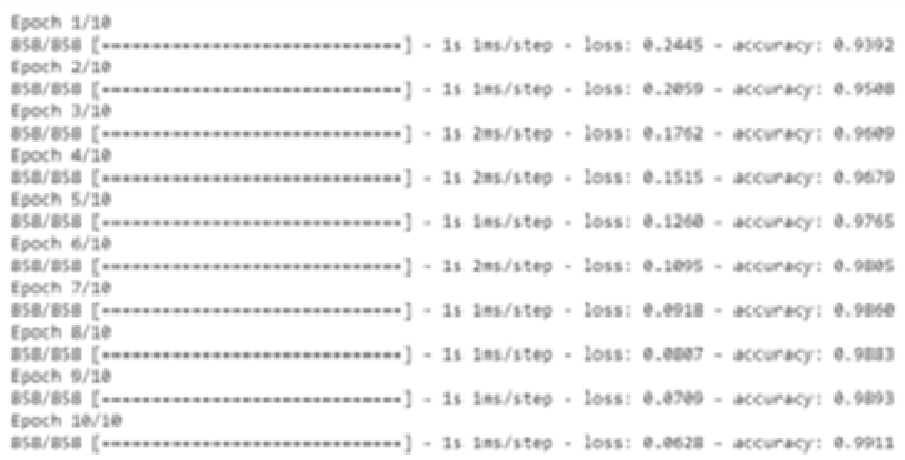

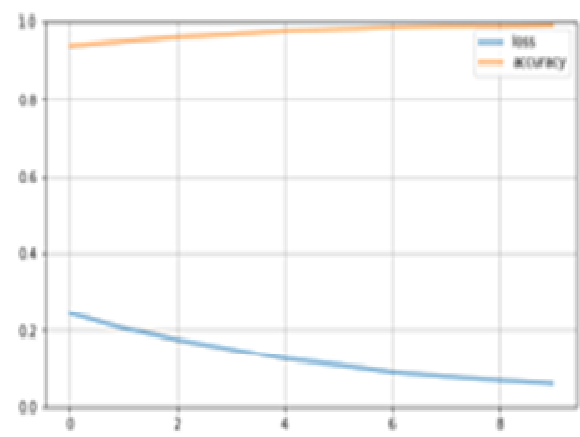

Figure 3: Convolution Neural Network Accuracy

\section{Conclusion and Future Scope}

Convolution neural network classifier shows $99.11 \%$ accuracy for proposed system. In this system different activation function experimented with different epoch. In this paper, convolution neural network is used for classification of hand gestures. In future, hand gesture classifies using feature convolution neural network and rectify accuracy of proposed system. For betterment of accuracy also use L1/L2 regularization and droop out concepts and avoid overfitting issue. 


\section{REFERENCES}

1. Adam Ahmed Qaid MOHAMMED, Jiancheng Lv and MD. Sajjatul Islam:A Deep Learning-Based End-to-End Composite System for Hand Detection and Gesture Recognition,Sensors 2019, 19, 5282; doi:10.3390/s19235282, www.mdpi.com/journal/sensors.

2. Mais Yasen and Shaidah Jusoh: A systematic review on hand gesture recognition techniques, challenges and Applications, PeerJ computer science, JusohDOI 10.7717/peerj-cs.2182019.

3. Paulo Trigueiros, Fernando Ribeiro et. al.:A comparison of machine learning algorithms applied to hand gesture recognition, researchgate, Paulo Trigueiros on 21 January 2014.

4. Wenjin Tao et. al:American Sign Language alphabet recognition using Convolutional Neural Networks with multiview augmentation and inference fusion, Artificial IntelligenceReceived 28 February 2018; Received in revised form 10 July 2018; Accepted 8 September 2018, 521976/@ 2018 Elsevier Ltd.

5. Dhir, S. P. (2018). Communication Network in Mimosa Pudica. International Journal of Botany and Research (IJBR), ISSN (P), $2277-4815$.

6. Jiachen YANG:An Improved Computer Interface Comprising a Recurrent Neural Network and a Natural User Interface, ScienceDirect, Procedia Computer Science 60 ( 2015 ) 1386 - 13951877-0509 @ 2015, Elsevier B.V, doi: 10.1016/j.procs.2015.08.213

7. Lionel Pigou: Beyond Temporal Pooling: Recurrence and Temporal Convolutions for Gesture Recognition in Video, February 11, 2016

8. Kenneth Lai et.al:CNN+RNN Depth and Skeleton based Dynamic Hand Gesture Recognition, 2018 24th International Conference on Pattern Recognition (ICPR) Beijing, China, August 20-24, 2018,978-1-5386-3788-3/18/\$31.00 (C2018 IEEE.

9. Debi Prosad Dogra et. al:Computer Vision Assisted Palm Rehabilitation With Supervised Learning, 10.1109/TBME.2015.2480881, IEEE Transactions on Biomedical Engineering.

10. Ketki Kshirsagar et. al.: Comparing Key Frame Selection for One-Two Hand Gesture Using Different Methods, International Journal of Signal and Imaging Systems Engineering, Int. J. Signal and Imaging Systems Engineering(2015), Vol. 8, No. 5, pp.273-285. Print ISSN: 17480698 Online ISSN: 1748-0701.

11. Kabrane, M., Krit, S., \& Elmaimouni, L. REDUCING TRAFFIC CONGESTION IN MODERN CITIES: A CHALLENGE BASED ON PHYSICO-MATHEMATICAL THEORIES AND WIRELESS SENSOR NETWORK.

12. Juan C. Núñez: Convolutional Neural Networks and Long Short-Term Memory for skeleton-based human activity and hand gesture recognition, 0031-3203/@ 2017 Elsevier Ltd.

13. Di Wu, Lionel Pigou: Deep Dynamic Neural Networks for Multimodal Gesture Segmentation and Recognition, IEEE Transactions on Pattern Analysis and Machine Intelligence, January 2016.

14. Cristina Nuzzi et. al.:Deep Learning-Based Hand Gesture Recognition for Collaborative Robots, IEEE Instrumentation \& Measurement MagazineApril 2019, 1094-6969/19/\$25.00@2019IEEE.

15. Lan, Y., YazaiXie, X. L., \& Zhang, W. THE MODEL OF INFORMATION PROPAGATION COMBINED WITH OPINION EVOLUTION ON SCALE-FREE NETWORK.

16. Guillaume Devineau, Wang Xi et. al.:Deep Learning for Hand Gesture Recognition on Skeletal Data, 13th IEEE Conference on Automatic Face and Gesture Recognition (FG'2018), May 2018, Xi'An, China.10.1109/FG.2018.00025.

17. Istvan Ketyko et. al.:Domain Adaptation for sEMG-based Gesture Recognition with Recurrent Neural Networks, Nokia Bell Labs@2019 IEEE, DOI: $10.1109 / I J C N N .2019 .8852018$

18. Kabrane, M., Krit, S., \& Elmaimouni, L. REDUCING TRAFFIC CONGESTION IN MODERN CITIES: A CHALLENGE BASED ON PHYSICO-MATHEMATICAL THEORIES AND WIRELESS SENSOR NETWORK.

19. Son Thai Ly et. al., "Emotion Recognition via Body Gesture: Deep Learning Model Coupled with Keyframe Selection”, MLMI2018, September 28-30, 2018, Ha Noi, Viet Nam, (C) 2018 Association for Computing Machinery. 
20. Kollipara Sai Varun et. al.:Hand Gesture Recognition and Implementation for Disables using CNN, International Conference on Communication and Signal Processing, April 4-6, 2019, India, 978-1-5386-7595-3/19/\$31.00 @2019 IEEE

21. KAUR, H., \& KAUR, H. DESIGN OF A CUSTOM ENCRYPTION KEY GENERATOR TO SECURE WIRELESS NETWORKS.

22. Peng Liu et. al.: Hand Gesture Recognition Based on Single-Shot Multibox Detector Deep Learning, Hindawi, Mobile Information Systems, Volume 2019, Article ID 3410348, P-7. 